\title{
Downsizing a Thrombus of Advanced Renal Cell Carcinoma in a Presurgical Setting with Sorafenib
}

\author{
Hirokazu Takeda ${ }^{a}$ Yojiro Nakano $^{a}$ Yuhta Kashiwagi $^{\mathrm{a}}$ Yasushi Yoshino $^{\mathrm{b}}$ \\ Momokazu Gotoh ${ }^{\text {b }}$

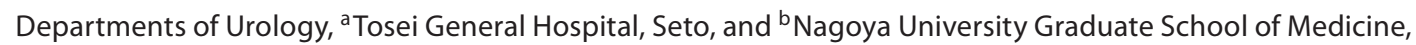 \\ Nagoya, Japan
}

\section{Key Words}

Presurgical setting $\cdot$ Sorafenib - Tumor thrombus •

Renal cancer

\begin{abstract}
A 53-year-old man presented with fever, $15 \mathrm{~kg}$ weight loss, ECOG performance status 0 , and $12 \times 9.5 \mathrm{~cm}$ renal tumor with an associated level II (near level III) tumor thrombus extending into the vena cava. We offered a presurgical targeted therapy to downsize the thrombus and primary tumor, which may reduce the extent of the surgery and operative risk. The patient accepted this approach with administration of sorafenib, resulting in a marked reduction of the primary renal tumor and $43 \%$ regression in tumor thrombus. Tumor shrinkage and regression of the thrombus allowed resection of the left kidney. Pathological findings revealed that part of the tumor was necrotic tissue. Two years after initiation of presurgical sorafenib therapy, the patient remains alive without evidence of disease progression.
\end{abstract}

Copyright $\odot 2011$ S. Karger AG, Basel

\section{KARGER}

Fax +4161306 1234

E-Mail karger@karger.ch

www.karger.com
(C) 2011 S. Karger AG, Basel

0042-1138/12/0882-0235\$38.00/0

Accessible online at:

www.karger.com/uin

\section{Introduction}

Upfront cytoreductive nephrectomy $(\mathrm{CN})$ followed by cytokine therapy has been shown to improve survival in patients with metastatic renal cell carcinoma (mRCC) $[1,2]$. However, some patients are not suitable candidates for $\mathrm{CN}$, metastasectomy, or thrombectomy owing to the extensive burden of disease. Recently, tyrosine kinase inhibitors (TKIs) have been used in the presurgical setting (targeted therapy prior to surgery) for the patients with primary RCC difficult to resect to facilitate surgery by reducing the tumor size [3-7]. Targeted therapy including sorafenib has been shown to reduce tumors of patients with mRCC compared with cytokine therapy. In phase II studies, sorafenib demonstrated a greater rate of tumor size reduction and better QOL compared with cytokine therapy [8]. The aim of this case report was to examine the clinical results of presurgical targeted therapy with sorafenib in a patient with unresectable mRCC. In addition, we analyzed the histopathological changes to determine the effects of sorafenib on tumor tissues. 

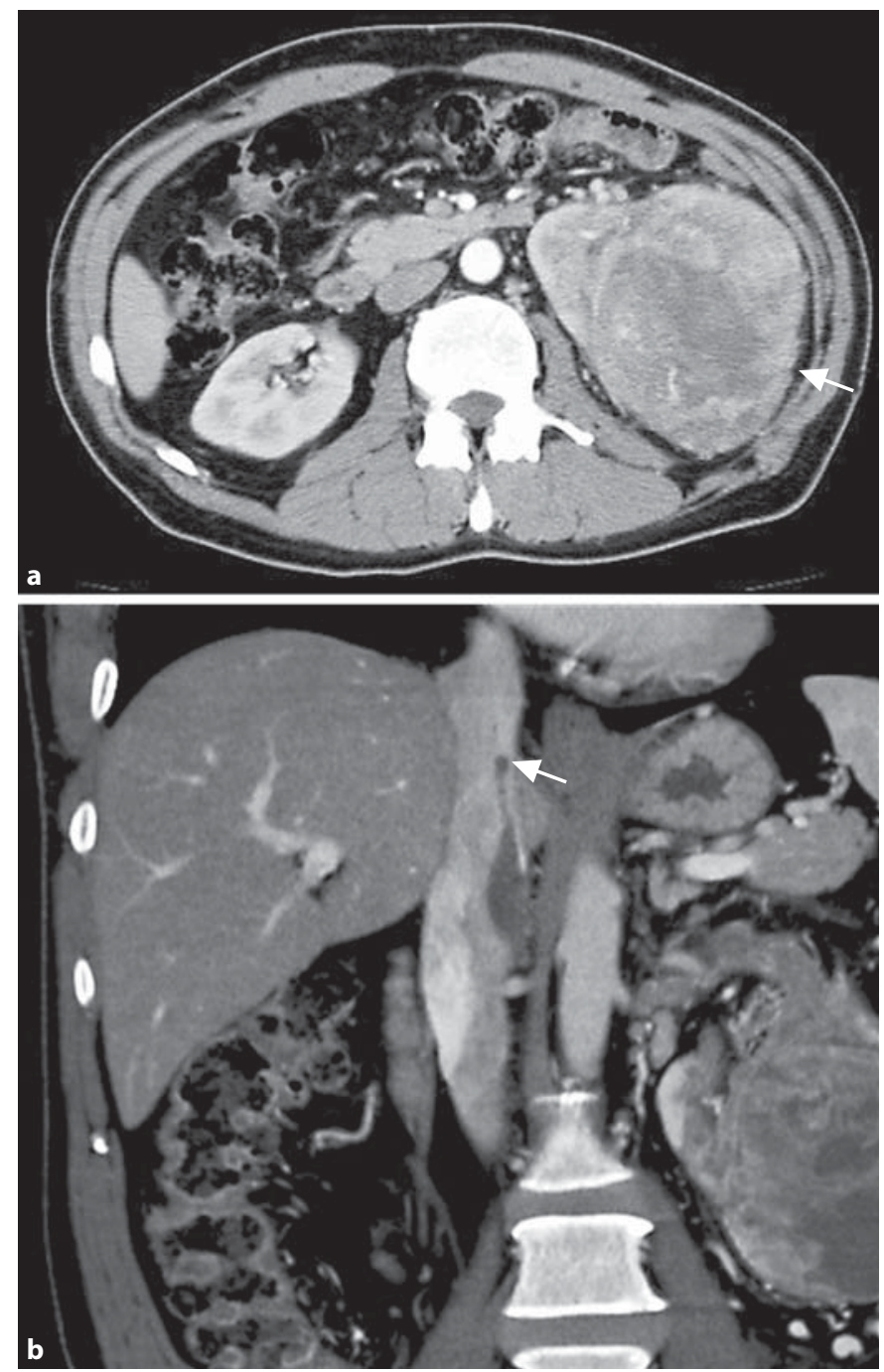

Fig. 1. Imaging of the patient's kidney before presurgical sorafenib therapy. a Primary left renal tumor measuring $12 \times 9.5 \mathrm{~cm}$ (arrow) seen on CT. $\mathbf{b}$ Tumor thrombus extending into the inferior vena cava (arrow) seen on CT.

\section{Case Report}

A 53-year-old man presented to a community hospital with a 6-month history of progressively worsening fever and $15-\mathrm{kg}$ weight loss. Serum C-reactive protein (CRP) elevated to $17.2 \mathrm{mg} /$ dl. His past medical history was unremarkable. Abdominal computed tomography (CT) revealed an enhanced tumor $(12 \times$ $9.5 \mathrm{~cm}$ ), which was clinically diagnosed as a left RCC associated with a level II (near level III) tumor thrombus, cT4N2M1, according to the tumor node metastasis (TNM) staging system [9] (fig. 1). European Cooperative Oncology Group (ECOG) performance status and MSKCC risk category were ECOG 1 and intermediate, respectively, and also multiple pulmonary metastases
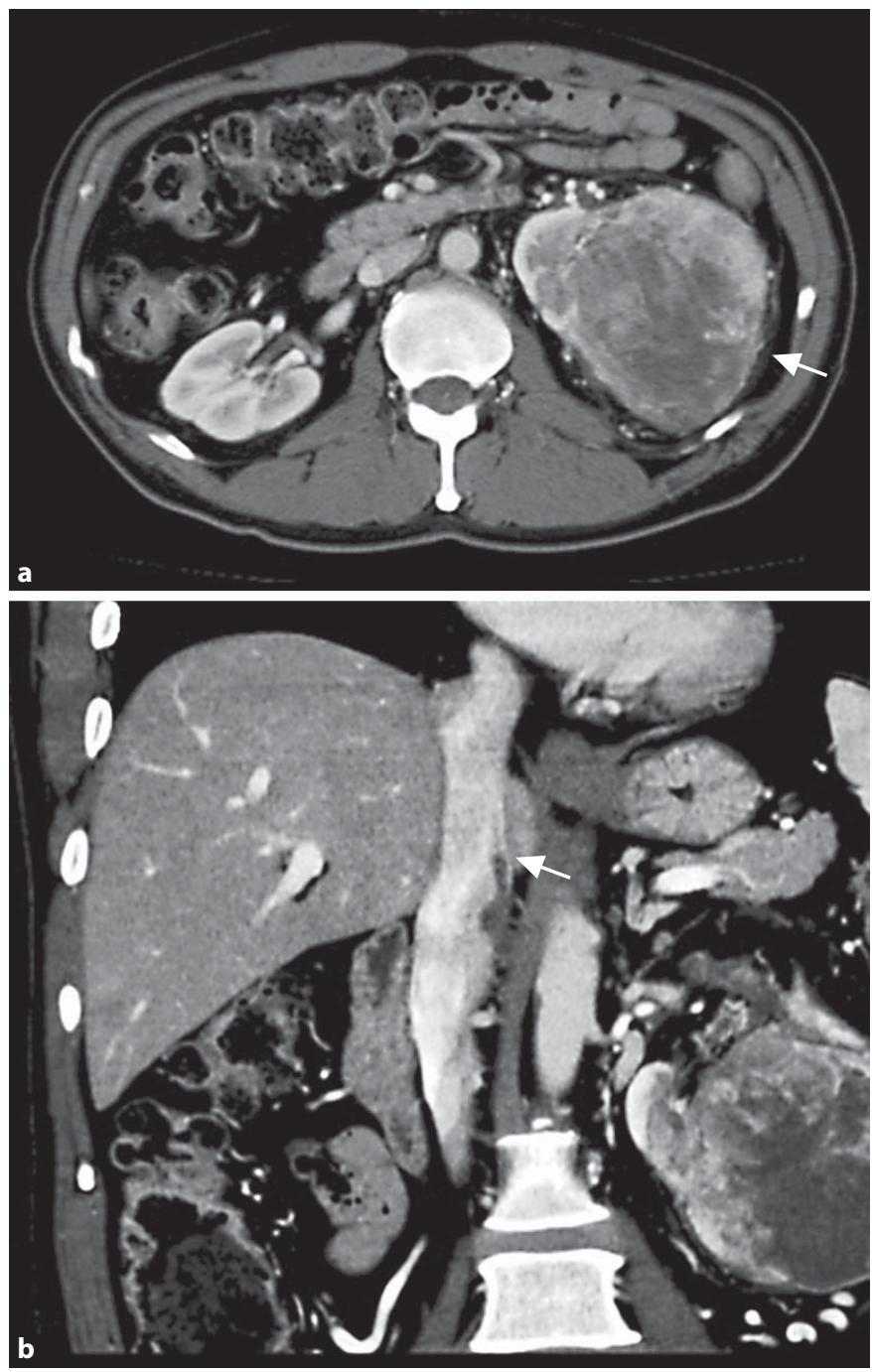

Fig. 2. Imaging of the patient's kidney after presurgical sorafenib therapy. a CT scan showed a reduction in the primary tumor of up to $11.4 \%$ (arrow). b Marked reduction in size of the tumor thrombus, which retracted well into the renal vein (arrow), seen on CT.

were detected. Treatment options were discussed with the patient, particularly, the use of targeted therapy to shrink the tumor to facilitate open left radical nephrectomy. The option of thrombus cytoreduction with sorafenib in the presurgical setting was offered. Starting in December 2008, sorafenib was administered at $400 \mathrm{mg}$ daily for 2 weeks, a lower dosage in consideration of an elevated CRP level and prolonged fever, followed by dose escalation to $400 \mathrm{mg}$ twice daily for 10 weeks. The dose was reduced to $400 \mathrm{mg}$ daily for 1 week in consideration of grade 2 hand-foot syndrome. Thirteen weeks after initiation of presurgical sorafenib therapy, CT scan showed a $43 \%$ regression of the inferior vena cava (IVC) thrombus as well as size reduction (11.4\%) of the primary tumor (fig. 2). Expected decrease of surgical invasion be- 
cause of partial imaging response and marked reduction of CRP levels $(1.7 \mathrm{mg} / \mathrm{dl})$ made the patient to accept the surgical treatment. Sorafenib was discontinued for 2 weeks followed by open left radical nephrectomy and IVC thrombectomy. Pathologic examination demonstrated pT4N2Mx, Fuhrman grade 2, clear cell RCC with extensive necrosis. No intra- or postoperative complications were observed, including wound healing complications or hemorrhage. Two weeks after surgery, sorafenib was restarted at $400 \mathrm{mg}$ twice daily for 6 months. Serum level of CRP was normalized $(0.20 \mathrm{mg} / \mathrm{dl})$ at 9 weeks after surgery. CT showed a shrinkage by up to approximately $20 \%$ in pulmonary metastasis. The patient was then switched to treatment with combination therapy of sorafenib (400 mg daily) and IFN $\alpha$ (3 MIU 3 times a week) for a year. Two years after initiation of presurgical sorafenib therapy, the patient remains alive without evidence of disease progression.

\section{Discussion}

Before the approval of targeted agents including sorafenib, the majority of patients with advanced RCC had received treatment with cytokines such as IFN $\alpha$ and IL-2. Upfront CN followed by cytokine therapy also has been shown to improve survival in patients with mRCC in the era of cytokine therapy. Cytokine therapy, however, is likely to be limited by its modest overall response rate and short intervals to progression. Moreover, some patients are not suitable candidates for $\mathrm{CN}$, metastasectomy, or thrombectomy owing to the extensive burden of disease. In the era of cytokine therapy, although one report showed the antitumor effect of neoadjuvant cyto- kine therapy including IFN $\alpha-2 b$, survival benefit was limited [10]. In the era of TKIs, the use of targeted therapies before surgery, including neoadjuvant therapy, induction therapy, and presurgical therapy for unresectable/resectable primary RCC, has been suggested as a treatment option, based on the available results of clinical trials of TKIs: two reports showed reduction of tumor size with a median of 14 and $24 \%$, respectively $[6,7]$. However, there is concern that minimal downsizing might not increase the surgical resectability of primary tumor. A recent report showed a significant reduction of thrombus extension in the IVC after presurgical sunitinib therapy [5]. These data suggest that downsizing the primary tumor with TKIs could be another option to increase surgical resectability. Similarly, in our case, sorafenib in the presurgical setting markedly shrunk the IVC thrombus; therefore, the complexity of open radical nephrectomy with vena caval thrombectomy was reduced to enable the resection with smaller invasion. No intra- or postoperative complications, including wound healing delay or hemorrhage accompanied the surgery. There was a possibility that the extensive necrosis in primary tumor was induced by sorafenib. In conclusion, this case study indicates that the IVC thrombus regressing effects of presurgical targeted therapy with sorafenib offers several potential advantages for patients with unresectable primary RCC. Sorafenib in the presurgical setting may afford the chance to render a difficult resection possible.

\section{References}

$>1$ Leibovich BC, Blute ML: Surgical management of renal cell carcinoma. Semin Oncol 2006;33:552-562.

$>2$ Wood CG: The role of cytoreductive nephrectomy in the management of metastatic renal cell carcinoma. Urol Clin North Am 2003;30:581-588.

73 Cowey CL, Amin C, Pruthi RS, et al: Neoadjuvant clinical trial with sorafenib for patients with stage II or higher renal cell carcinoma. J Clin Oncol 2010;28:1502-1507.

4 Shuch B, Riggs SB, LaRochelle JC, et al: Neoadjuvant targeted therapy and advanced kidney cancer: observations and implications for a new treatment paradigm. BJU Int 2008; 102:692-696.
5 Harshman LC, Srinivas S, Kamaya A, Chung BI: Laparoscopic radical nephrectomy after shrinkage of a caval tumor thrombus with sunitinib. Nat Rev Urol 2009;6:338-343.

6 Bex A, van der Veldt AA, Blank C, et al: Neoadjuvant sunitinib for surgically complex advanced renal cell cancer of doubtful resectability: initial experience with downsizing to reconsider cytoreductive surgery. World J Urol 2009;27:533-539.

7 Thomas AA, Rini BI, Lane BR, et al: Response of the primary tumor to neoadjuvant sunitinib in patients with advanced renal cell carcinoma. J Urol 2009;181:518-523.
8 Escudier B, Szczylik C, Hutson TE, Demkow T, Staehler M, Rolland F, Negrier S, Laferriere N, Scheuring UJ, Cella D, Shah S, Bukowski RM. Randomized phase II trial of first-line treatment with sorafenib versus interferon Alfa-2a in patients with metastatic renal cell carcinoma. J Clin Oncol 2009;27: 1280-1289.

9 Sobin LH: TNM Classification of Malignant Tumors. Oxford, Wiley-Blackwell, 2009.

10 Bex A, Horenblas S, de Gast GC: The timing of immunotherapy and nephrectomy in multimodality treatment of metastatic renal cell carcinoma. Technol Cancer Res Treat 2003;2:205-210. 